Review Article

\title{
Effectiveness of Hyaluronic Acid Administration in Treating Adhesive Capsulitis of the Shoulder: A Systematic Review of Randomized Controlled Trials
}

\author{
Lin-Chien Lee, ${ }^{1}$ Fu-Kong Lieu, ${ }^{1}$ Hung-Lin Lee, ${ }^{2}$ and Tao-Hsin Tung ${ }^{3,4}$ \\ ${ }^{1}$ Department of Physical Medicine and Rehabilitation, Cheng Hsin General Hospital, Taipei 11220, Taiwan \\ ${ }^{2}$ Department of Nursing, College of Medicine, National Taiwan University, Taipei 10051, Taiwan \\ ${ }^{3}$ Department of Medical Research and Education, Cheng Hsin General Hospital, Taipei 11220, Taiwan \\ ${ }^{4}$ Department of Public Health, School of Medicine, Fu Jen Catholic University, Taipei 24205, Taiwan
}

Correspondence should be addressed to Tao-Hsin Tung; ch2876@gmail.com

Received 11 August 2014; Accepted 18 January 2015

Academic Editor: Konstantinos Anagnostakos

Copyright (C) 2015 Lin-Chien Lee et al. This is an open access article distributed under the Creative Commons Attribution License, which permits unrestricted use, distribution, and reproduction in any medium, provided the original work is properly cited.

\begin{abstract}
Introduction. Adhesive capsulitis (AC) of the shoulder presents with an insidious onset of pain and progressive limitation of shoulder movement. Objectives. To investigate whether intra-articular hyaluronic acid (HA) administration alone is superior to conventional therapies and whether the addition of intra-articular HA administration to conventional therapies improves clinical outcomes in patients with AC. Methods. The PubMed, EMBASE, CINAHL, and Cochrane Library electronic databases were searched without language restrictions in July 2014 with a priori defined inclusion and exclusion criteria. Results. Four randomized controlled trials (273 participants, 278 shoulders) were included in this review. Two trials compared intra-articular HA administration with conventional therapies and 2 trials evaluated intra-articular HA administration as an addition to conventional therapies. Pain and shoulder function/disability outcomes in the HA injection group were not superior to those achieved in the conventional therapy groups. No significant differences in pain or shoulder function/disability outcomes were noted between the groups with and without adjunctive HA administration. Conclusions. Intra-articular HA administration alone is not superior to conventional AC treatments, and the addition of intra-articular HA administration to conventional therapies does not provide significant added benefits. HA administration in AC patients who are receiving conventional therapies should be evaluated to avoid unnecessary medical expenditure.
\end{abstract}

\section{Introduction}

Adhesive capsulitis (AC) of the shoulder manifests as an insidious onset of shoulder pain and the progressive limitation of active and passive shoulder movement, resulting in a marked disability and difficulty with daily activities. AC occurs in $2-5 \%$ of the general population, and predominantly in females. The age of onset of AC ranges from 40 to 60 years. The etiology and pathogenesis of primary AC remain largely unknown, but idiopathic inflammation in the lining of the shoulder joint is postulated to be the primary cause $[1,2]$. $\mathrm{AC}$ is associated with a variety of diseases, including diabetes mellitus, thyroid dysfunction, and autoimmune diseases, as well as a history of breast cancer treatment, stroke, or myocardial infarction [3]. The natural course of primary AC is not well established, and the clinical diagnosis is based on patient history and physical examination [4].

Treatment of AC aims to relieve pain and restore shoulder motion and function. Conventional therapies for $\mathrm{AC}$ include the use of analgesics and nonsteroidal antiinflammatory drugs, intra-articular corticosteroid administration, and physical therapy [4]. Treatment programs for AC usually combine a number of the aforementioned modalities to address the various symptoms and to achieve maximal outcomes [5]. In patients refractory to conventional therapies, more aggressive interventions such as capsular distention, manipulation under anesthesia, and surgery have been used [3]. 
Hyaluronic acid (HA) is an integral component of the synovial fluid and is crucial for the lubrication and chondroprotection of joints. By injecting HA into joints, cytokineinduced responses are suppressed and synovial inflammation is reduced, alleviating pain and improving joint function $[6,7]$. Furthermore, when HA is injected intra-articularly to treat $\mathrm{AC}$, the degree of suppressed inflammation is directly associated with the improvement in shoulder function [8]. The effectiveness of viscosupplementation with HA injection has been confirmed in the treatment of knee osteoarthritis $[9,10]$. However, the utility of HA administration in the treatment of AC is not universally endorsed, because of controversial evidence [11-13]. The objectives of our review were to investigate whether HA administration alone is superior to conventional AC therapies (e.g., intra-articular corticosteroid injection and physical therapy) and whether the addition of HA administration to conventional therapy regimens improves clinical outcomes in patients with AC.

\section{Methods}

2.1. Review Protocol. Our methodology and reporting were guided by the Preferred Reporting Items for Systematic Reviews and Meta-Analyses (PRISMA) statement and checklist [14]. This systematic review was accepted by the online PROSPERO international prospective register of systematic reviews of the National Institute for Health Research (CRD42014010363).

2.2. Data Sources and Searches. We systematically searched PubMed, EMBASE, CINAHL, and the Cochrane Library without language restrictions in July 2014. We entered "adhesive capsulitis," "frozen shoulder," "stiff shoulder," "periarthritis," "Duplay's disease," "hyaluronic acid," "sodium hyaluronate," and "viscosupplementation" as keywords for searches. We also searched reference lists of retrieved trials and contacted known experts in the field for potentially relevant studies not identified by the internet-based search. Unpublished trials were searched using the ClinicalTrials.gov registry (https://clinicaltrials.gov/).

2.3. Study Selection. Randomized controlled trials that directly compared HA injection (into the glenohumeral joint or the subacromial bursa) with conventional therapies (intraarticular corticosteroid injection or physical therapy) or that investigated the effectiveness of HA administration as an added treatment were eligible for inclusion. Prospective uncontrolled studies were excluded. Included trials must have clearly documented the inclusion and exclusion criteria for patient selection and information regarding HA administration. The symptoms of AC (pain, limited range of motion (ROM), and disability of the shoulder) must have persisted in patients for more than 1 month before trial registration. The diagnosis of AC must have been confirmed by clinical and/or ultrasonographic assessments. Quantitative assessments of shoulder pain, ROM, or function/disability before and following HA injections were reported in the included trials. A minimum of 1 month of follow-up was required.

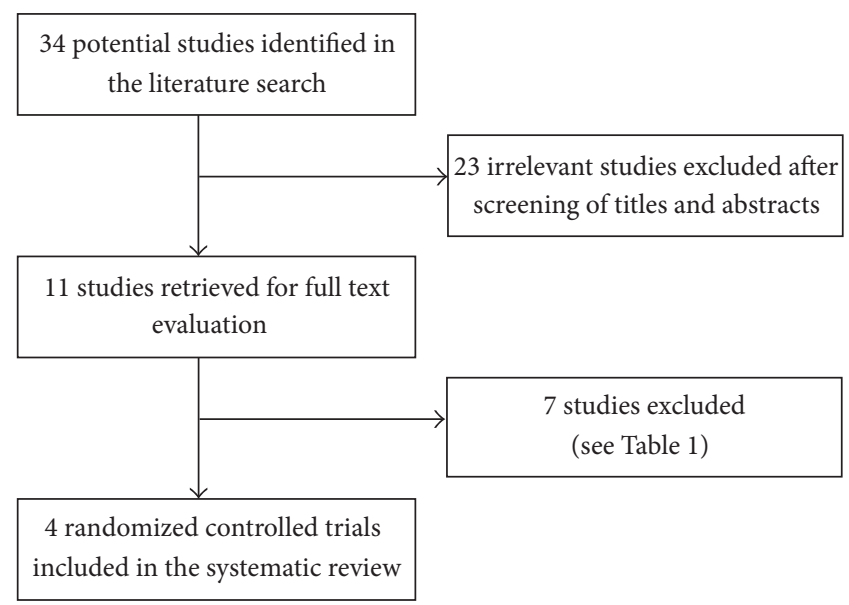

FIGURE 1: Flow diagram of the evaluation process for the studies included in the systematic review.

Trials that evaluated the treatment of causes of shoulder pain other than primary AC were excluded. When papers with overlapping data sets were published, the trial with the larger population was included.

2.4. Data Extraction and Quality Assessment. One author (Lin-Chien Lee) evaluated all potential studies eligible for inclusion and used a standardized form to extract data, including study population characteristics, study design, inclusion and exclusion criteria, information regarding HA administration, outcome measurements, and frequency of adverse events. A second author (Fu-Kong Lieu) verified the accuracy and completeness of the extracted data. The authors of included trials were contacted if additional information was necessary. Two authors (Lin-Chien Lee and Fu-Kong Lieu) independently evaluated the methodological quality of the included studies. The following domains were assessed: allocation generation and concealment, blinding, duration of follow-up, numbers of subjects not included in follow-up, and data analysis method (intention-to-treat or per protocol). Discrepancies were resolved by discussion and consensus.

2.5. Data Synthesis and Analysis. The outcomes of effectiveness were protocol-defined pain score, ROM, and shoulder function/disability scores at postinjection follow-up assessments more than 1 month after administration. Adverse events were also a main outcome of interest. The included trials were summarized qualitatively. We quantitatively combined trials using meta-analysis if the study designs were sufficiently similar with regard to the study population, interventions being compared, and measured outcomes.

\section{Results}

3.1. Characteristics of the Included Trials. Thirty-four nonduplicate studies were identified in the literature search, and 11 full text reports of clinical trials were retrieved and screened for eligibility (Figure 1). One observational study not involving treatment [15] and 3 prospective uncontrolled 
TABLE 1: Detailed reasons for exclusion of individual studies.

\begin{tabular}{lcl}
\hline Author & Year & Reasons for exclusion \\
\hline Leardini et al. [16] & 1988 & $\begin{array}{l}\text { Non-RCT (prospective uncontrolled study) } \\
\text { Mixed diagnosis in the experimental group (OA, Duplay's disease, combined OA and Duplay's disease) } \\
\text { Follow-up duration shorter than 1 month (11 days) }\end{array}$ \\
\hline $\begin{array}{l}\text { Itokazu and } \\
\text { Matsunaga [17] }\end{array}$ & 1995 & $\begin{array}{l}\text { Non-RCT (prospective uncontrolled study) } \\
\text { Mixed diagnosis in the experimental group (subacromial bursitis, tendinitis, and capsulitis) } \\
\text { Duration of symptoms shorter than 1 month in some subjects }\end{array}$ \\
\hline $\begin{array}{l}\text { Tamai and Yamato } \\
\text { [15] }\end{array}$ & 1997 & Non-RCT (observational study) \\
\hline Tamai et al. [18] & 1999 & $\begin{array}{l}\text { Follow-up duration shorter than 1 month (7 days) } \\
\text { Primary intention was not treatment of AC } \\
\text { Participants in the AC group were the same as Tamai et al., 2004 [8] }\end{array}$ \\
\hline Tamai et al. [8] & 2004 & $\begin{array}{l}\text { Non-RCT (prospective uncontrolled study) } \\
\text { Follow-up duration shorter than 1 month (1 week) } \\
\text { Primary intention was not treatment of AC }\end{array}$ \\
\hline Blaine et al. [19] & 2008 & $\begin{array}{l}\text { Mixed diagnosis in the experimental group (OA, rotator cuff tear, and/or AC) } \\
\text { Lee et al. [20] }\end{array}$ \\
\hline AC: adhesive capsulitis; OA: osteoarthritis; RCT: randomized controlled trial. \\
\hline
\end{tabular}

studies were excluded $[8,16,17]$. One study was excluded because of a postinjection follow-up interval shorter than 1 month (7 days) [18]. One study was excluded because the study participants included shoulder osteoarthritis and rotator cuff tear patients in addition to those with AC [19]. One study was excluded because the authors combined corticosteroid and HA injections in the treatment course (1 dose of corticosteroid followed by 5 weekly doses of HA), and pain reduction effects and functional improvements could have been caused by the corticosteroid, HA, or both [20]. Detailed reasons for the exclusion of studies are summarized in Table 1.

Four trials with variable methodological quality met our inclusion criteria, all of which had been published as peer-reviewed articles. Study population characteristics and experimental design information for each of the 4 trials included in this review are shown in Table 2 . The number of participants in the included trials ranged from 30 to 90 , and 273 participants (278 shoulders) were included in our systematic review. Mean ages ranged from 54.5 to 64.2 years. The duration from the onset of AC symptoms to trial registration ranged from 1 to 8.3 months. Musculoskeletal ultrasonography was used to confirm the diagnosis of $\mathrm{AC}$ in 2 of the 4 included trials $[21,22]$. In 3 trials, HA was administered intra-articularly under landmark guidance [12, $13,21]$, and 1 trial was performed under ultrasonographyguided injection [22]. The type of HA, number and dosage of HA injections, and postinjection follow-up duration varied widely between trials.

Two trials evaluated the added effect of intra-articular HA injections to conventional therapies $[13,21]$, and the other 2 compared intra-articular HA injection with conventional therapies $[12,22]$. In the reviewed studies, the Visual Analogue Scale, the Verbal Numeric Scale, and the pain subscale of the Shoulder Pain and Disability Index were used as pain measures, and the Constant Score, the Shoulder Pain and
Disability Index, and the Shoulder Disability Questionnaire were used as function/disability measures. Measurements for ROM included abduction, flexion, internal rotation, and external rotation.

3.2. Risk of Bias Assessments. The methodological quality of the included trials is shown in Table 3. The most common sources of potential bias were inadequate allocation generation and concealment. We did not combine the included trials using meta-analysis methods, because the number of included trials was small and heterogeneity existed in the study designs, interventions being compared (head-to-head comparison or as an added treatment), regimens, and preparations (molecular weight and viscosity) of $\mathrm{HA}$, as well as in the outcomes of interest among the included trials.

3.3. HA versus Conventional Therapies: Pain, Function/Disability, and ROM. Two trials investigated the effectiveness of intra-articular HA injection as compared with conventional therapies [12, 22]. Calis et al. [12] divided participants into 4 groups and compared the effectiveness of intra-articular corticosteroid injection, intra-articular HA injection, physical therapy, and home exercise. Reported outcomes included shoulder pain (Visual Analogue Scale), function/disability (Constant Score), and ROM (passive) of the shoulder. The only significant difference observed in any measured outcome among the corticosteroid, HA, and physical therapy groups at 3 months after injection was significantly improved passive external rotation in the physical therapy group in comparison to the corticosteroid and HA groups. Park et al. [22] evaluated intra-articular HA administration with capsular distention (achieved by $0.5 \%$ lidocaine, $18 \mathrm{~mL}$ ) in comparison to intra-articular corticosteroid injection. Reported outcomes included pain (Verbal Numeric Scale), function/disability (Shoulder Pain and Disability Index), and ROM (passive). The only significant difference that was 


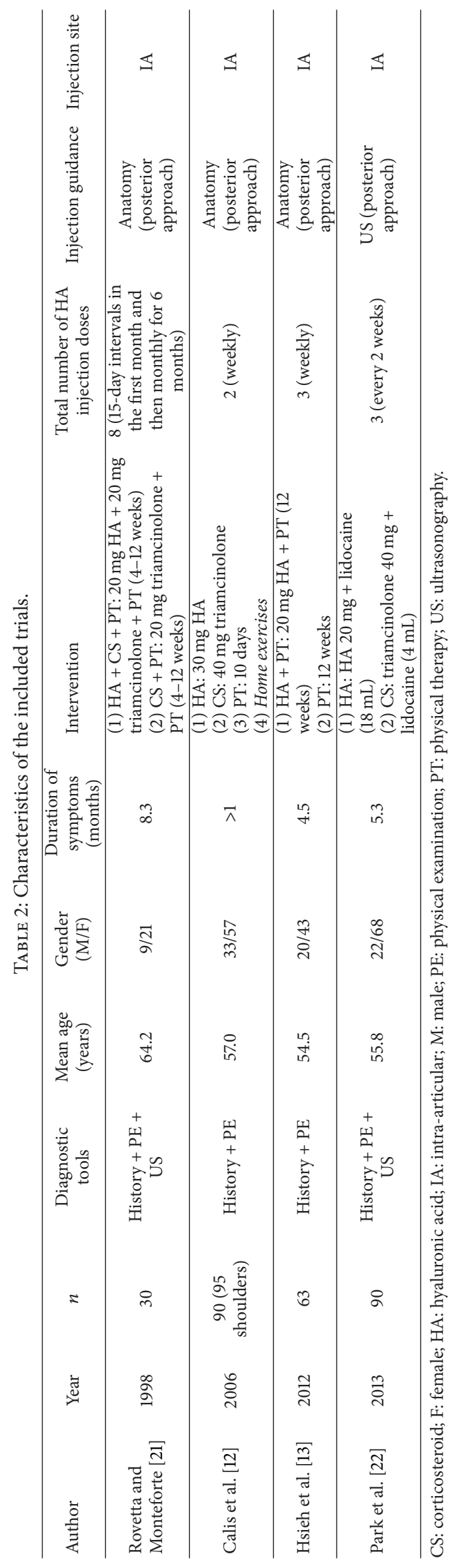




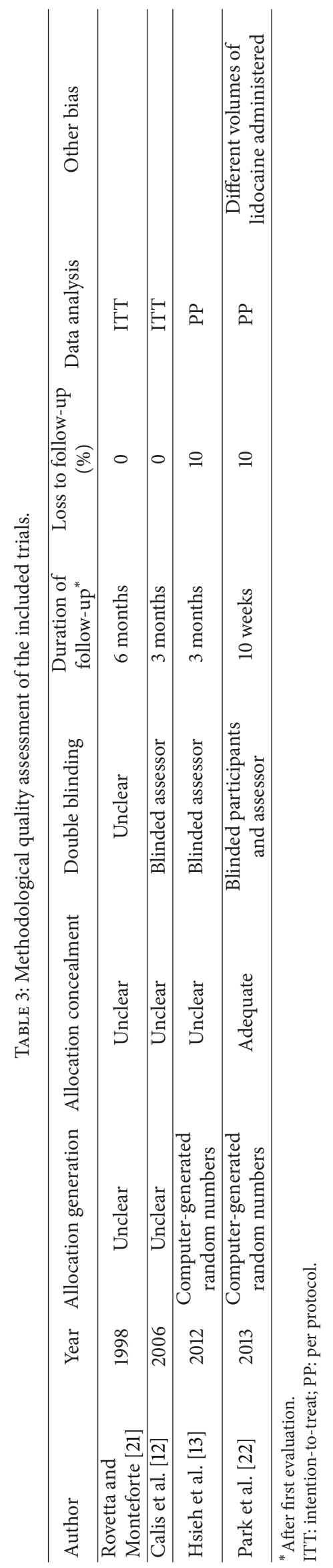


obtained in any measured outcome at 10 weeks after injection was that passive external rotation was significantly improved in the HA (combined with capsular distention) group.

3.4. HA as Adjunct Treatment: Pain, Function/Disability, and $R O M$. Two trials investigated the effectiveness of intraarticular HA administration as an adjunct therapy in the treatment of patients with AC $[13,21]$. Rovetta and Monteforte [21] compared combination therapy using intraarticular corticosteroid/HA administration and physical therapy to combination therapy using intra-articular corticosteroid injection and physical therapy. Reported outcomes included pain (Visual Analogue Scale) and ROM of the shoulder. No between-group comparison was performed, although both groups showed improvement of pain and ROM at 6 months after injection. They also observed that internal rotation was improved to a greater degree in the group that received intra-articular HA administration. Hsieh et al. [13] evaluated combination therapy using intra-articular HA injection and physical therapy in comparison to physical therapy alone. The reported outcomes included pain (pain subscale of the Shoulder Pain and Disability Index), function/disability (total scores of the Shoulder Pain and Disability Index and the Shoulder Disability Questionnaire), and ROM (passive and active). No significant difference was obtained in any measured outcome at 3 months after injection.

3.5. Adverse Events. Rovetta and Monteforte reported no major adverse events [21]. Park et al. reported that 12 out of 45 participants undergoing capsular distension combined with intra-articular HA injection experienced pain during the procedure, and the pain may be a result of the capsular distension procedure, the intra-articular HA injection, or both [22]. The adverse event data could not be obtained for the other 2 included trials $[12,13]$.

\section{Discussion}

Our review included 4 trials and provided a synthesis of the current evidence on intra-articular HA administration for the treatment of AC. Two trials investigated the effectiveness of intra-articular HA administration in comparison with conventional therapies, and the other 2 investigated the effectiveness of intra-articular HA administration as an adjunct therapy. The scopes of these reports are reflective of clinical practice. Intra-articular HA administration is not superior to conventional therapies (intra-articular corticosteroid administration and physical therapy) for the treatment of patients with AC, and the addition of intra-articular HA administration to conventional therapies is not expected to provide significant added benefits. Thus, the potential role of $\mathrm{HA}$ in the treatment of AC remains controversial.

In short-term follow-up assessments less than 3 months after administration, 2 of the included trials showed that the clinical effectiveness of intra-articular HA administration in terms of pain and shoulder function/disability was not superior to corticosteroid administration [12, 22], and this conclusion is also supported by a recent systematic review
[11]. Intra-articular injections of either corticosteroids or HA reduce inflammation and pain in patients with $\mathrm{AC}$ and lead to functional improvement. The onset of the effects of corticosteroid administration is faster than HA administration, but the effects of HA may last longer than those of corticosteroids $[10,23]$. Intra-articular administration of corticosteroids for the treatment of AC may be beneficial, although their effects may be relatively weak and only of short-term benefit $[4,24$, 25]. Intra-articular administration of HA is more costly than corticosteroids for the treatment of AC, but fewer adverse events have been associated with $\mathrm{HA}$ administration [11]. In our review, the long-term effects ( $>6$ months) of HA injection for the treatment of $\mathrm{AC}$ could not be ascertained because the included trials did not conduct long-term follow-up assessments.

Although the benefit of physical therapy in the treatment of AC is not confirmed by systematic reviews [26, 27], some clinical studies with lower grades of evidence have reported its benefits, and it is commonly used in AC treatment [3]. Calis et al. concluded that physical therapy provided clinical benefits superior to intra-articular HA and corticosteroid administrations [12]. Hsieh et al. also found significant improvements in patients treated with physical therapy [13]. Accordingly, physical therapy can be considered as a treatment option in patients with AC.

Rovetta and Monteforte administered corticosteroids alone or in combination with HA to treat AC [21]. Both treatment groups showed improvements in pain and ROM after 6 months of treatment, but the added benefit of HA to corticosteroid administration was not confirmed. Furthermore, Hsieh et al. showed that HA administration did not provide significant added benefits for patients with AC who were already receiving regular physical therapy [13]. Intra-articular HA administration for patients with AC who are already receiving regular conventional therapies should be carefully evaluated to avoid unnecessary medical expenditure.

Although the majority of the reviewed studies (3 of 4) used landmark-guided injection, the negative results of our review might not be attributable to the type of injection guidance (landmark or ultrasonography-guided). Lee et al. showed that the clinical effectiveness of ultrasonographyguided injection (1 initial corticosteroid dose, followed by 5 weekly doses of HA) for AC was not superior to landmarkguided injection after the first few weeks of treatment [20]. Similarly, ultrasonography guidance may improve the accuracy of corticosteroid injection for the treatment of shoulder pain, but clinical advantages of ultrasonography-guided injection over landmark-guided injection in terms of pain, function, ROM, and adverse events were not supported by the evidence $[28,29]$.

Our review differs from a previous review published in 2011 [11], because our review includes only randomized controlled trials, considers only studies with participants diagnosed with AC, reports shoulder pain outcomes in addition to function/disability and ROM, and focuses on the role of intra-articular HA administration compared to conventional therapies and role of $\mathrm{HA}$ administration as an 
added treatment. We also have included 2 trials that were published since $2011[13,22]$.

Several limitations of this review should be addressed. First, the small number of included trials limits the strength of the conclusions that were drawn. Second, the included trials are of variable methodological quality, which introduced the risk of bias (Table 3 ). Third, the participants recruited in our reviewed trials may differ with regard to the stage and severity of AC, and this may have limited our ability to measure beneficial effects of HA use in this review. Fourth, the majority of reviewed trials arranged follow-up assessments for 3-6 months after treatment, and thus the long-term effects of HA administration were not evaluated.

\section{Conclusions}

Intra-articular HA administration alone is not superior to conventional AC treatments, and the addition of intraarticular HA administration to conventional therapies does not provide significant added benefits. AC can be divided into 4 consecutive stages [3], and further randomized controlled studies involving large sample sizes and stage-based designs (analysis stratified by the stages of AC) are needed to verify the effectiveness of HA administration when used alone or in combination with other conventional therapies.

\section{Conflict of Interests}

The authors declare that there is no conflict of interests regarding the publication of this paper.

\section{References}

[1] R. J. Neviaser and T. J. Neviaser, "The frozen shoulder diagnosis and management," Clinical Orthopaedics and Related Research, no. 223 , pp. 59-64, 1987.

[2] S. A. Rodeo, J. A. Hannafin, J. Tom, R. F. Warren, and T. L. Wickiewicz, "Immunolocalization of cytokines and their receptors in adhesive capsulitis of the shoulder," Journal of Orthopaedic Research, vol. 15, no. 3, pp. 427-436, 1997.

[3] A. S. Neviaser and J. A. Hannafin, "Adhesive capsulitis: a review of current treatment," The American Journal of Sports Medicine, vol. 38, no. 11, pp. 2346-2356, 2010.

[4] M. J. Griesser, J. D. Harris, J. E. Campbell, and G. L. Jones, "Adhesive capsulitis of the shoulder: a systematic review of the effectiveness of intra-articular corticosteroid injections," The Journal of Bone and Joint Surgery. American Volume, vol. 93, no. 18, pp. 1727-1733, 2011.

[5] V. Blanchard, S. Barr, and F. L. Cerisola, "The effectiveness of corticosteroid injections compared with physiotherapeutic interventions for adhesive capsulitis: a systematic review," Physiotherapy, vol. 96, no. 2, pp. 95-107, 2010.

[6] H. Iwata, "Pharmacologic and clinical aspects of intraarticular injection of hyaluronate," Clinical Orthopaedics and Related Research, no. 289, pp. 285-291, 1993.

[7] D. D. Waddell, O. V. Kolomytkin, S. Dunn, and A. A. Marino, "Hyaluronan suppresses IL-1 $\beta$-induced metalloproteinase activity from synovial tissue," Clinical Orthopaedics and Related Research, no. 465, pp. 241-248, 2007.
[8] K. Tamai, H. Mashitori, W. Ohno, J. Hamada, H. Sakai, and K. Saotome, "Synovial response to intraarticular injections of hyaluronate in frozen shoulder: a quantitative assessment with dynamic magnetic resonance imaging," Journal of Orthopaedic Science, vol. 9, no. 3, pp. 230-234, 2004.

[9] R. R. Bannuru, N. S. Natov, U. R. Dasi, C. H. Schmid, and T. E. McAlindon, "Therapeutic trajectory following intra-articular hyaluronic acid injection in knee osteoarthritis-metaanalysis," Osteoarthritis and Cartilage, vol. 19, no. 6, pp. 611-619, 2011.

[10] N. Bellamy, J. Campbell, V. Robinson, T. Gee, R. Bourne, and G. Wells, "Viscosupplementation for the treatment of osteoarthritis of the knee," Cochrane Database of Systematic Reviews, no. 2, Article ID CD005321, 2006.

[11] J. D. Harris, M. J. Griesser, A. Copelan, and G. L. Jones, “Treatment of adhesive capsulitis with intra-articular hyaluronate: a systematic review," International Journal of Shoulder Surgery, vol. 5, no. 2, pp. 31-37, 2011.

[12] M. Calis, H. Demir, S. Ulker, M. Kirnap, F. Duygulu, and H. T. Calis, "Is intraarticular sodium hyaluronate injection an alternative treatment in patients with adhesive capsulitis?" Rheumatology International, vol. 26, no. 6, pp. 536-540, 2006.

[13] L.-F. Hsieh, W.-C. Hsu, Y.-J. Lin, H.-L. Chang, C.-C. Chen, and V. Huang, "Addition of intra-articular hyaluronate injection to physical therapy program produces no extra benefits in patients with adhesive capsulitis of the shoulder: a randomized controlled trial," Archives of Physical Medicine and Rehabilitation, vol. 93, no. 6, pp. 957-964, 2012.

[14] A. Liberati, D. G. Altman, J. Tetzlaff et al., "The PRISMA statement for reporting systematic reviews and meta-analyses of studies that evaluate health care interventions: explanation and elaboration," Journal of Clinical Epidemiology, vol. 62, no. 10, pp. el-e34, 2009.

[15] K. Tamai and M. Yamato, "Abnormal synovium in the frozen shoulder: a preliminary report with dynamic magnetic resonance imaging," Journal of Shoulder and Elbow Surgery, vol. 6, no. 6, pp. 534-543, 1997.

[16] G. Leardini, A. Perbellini, M. Franceschini, and L. Mattara, "Intra-articular injections of hyaluronic acid in the treatment of painful shoulder," Clinical Therapeutics, vol. 10, no. 5, pp. 521526, 1988.

[17] M. Itokazu and T. Matsunaga, "Clinical evaluation of highmolecular-weight sodium hyaluronate for the treatment of patients with periarthritis of the shoulder," Clinical Therapeutics, vol. 17, no. 5, pp. 946-955, 1995.

[18] K. Tamai, M. Yamato, J. Hamada, H. Mashitori, and K. Saotome, "Response of frozen shoulder to intraarticular corticosteroid and hyaluronate: a quantitative assessment with dynamic magnetic resonance imaging," Dokkyo Journal of Medical Sciences, vol. 26, no. 2, pp. 235-241, 1999.

[19] T. Blaine, R. Moskowitz, J. Udell et al., "Treatment of persistent shoulder pain with sodium hyaluronate: a randomized, controlled trial. A multicenter study," The Journal of Bone and Joint Surgery-American volume, vol. 90, no. 5, pp. 970-979, 2008.

[20] H.-J. Lee, K.-B. Lim, D.-Y. Kim, and K.-T. Lee, "Randomized controlled trial for efficacy of intra-articular injection for adhesive capsulitis: ultrasonography-guided versus blind technique," Archives of Physical Medicine and Rehabilitation, vol. 90, no. 12, pp. 1997-2002, 2009. 
[21] G. Rovetta and P. Monteforte, "Intraarticular injection of sodium hyaluronate plus steroid versus steroid in adhesive capsulitis of the shoulder," International Journal of Tissue Reactions, vol. 20, no. 4, pp. 125-130, 1998.

[22] K. D. Park, H.-S. Nam, J. K. Lee, Y. J. Kim, and Y. Park, "Treatment effects of ultrasound-guided capsular distension with hyaluronic acid in adhesive capsulitis of the shoulder," Archives of Physical Medicine and Rehabilitation, vol. 94, no. 2, pp. 264-270, 2013.

[23] N. Bellamy, J. Campbell, V. Robinson, T. Gee, R. Bourne, and G. Wells, "Intraarticular corticosteroid for treatment of osteoarthritis of the knee," Cochrane Database of Systematic Reviews, no. 2, Article ID CD005328, 2006.

[24] R. Buchbinder, S. Green, and J. M. Youd, "Corticosteroid injections for shoulder pain," The Cochrane Database of Systematic Reviews, no. 1, Article ID CD004016, 2003.

[25] A. Bal, E. Eksioglu, B. Gulec et al., "Effectiveness of corticosteroid injection in adhesive capsulitis," Clinical Rehabilitation, vol. 22, no. 6, pp. 503-512, 2008.

[26] S. Green, R. Buchbinder, and S. Hetrick, "Physiotherapy interventions for shoulder pain," The Cochrane Database of Systematic Reviews, no. 2, Article ID CD004258, 2003.

[27] M. J. Page, S. Green, S. Kramer et al., "Manual therapy and exercise for adhesive capsulitis (frozen shoulder)," The Cochrane Database of Systematic Reviews, no. 8, Article ID CD011275, 2014.

[28] J. E. Bloom, A. Rischin, R. V. Johnston, and R. Buchbinder, "Image-guided versus blind glucocorticoid injection for shoulder pain," The Cochrane Database of Systematic Reviews, no. 8, Article ID CD009147, 2012.

[29] W. Sage, L. Pickup, T. O. Smith, E. R. E. Denton, and A. P. Toms, "The clinical and functional outcomes of ultrasoundguided vs landmark-guided injections for adults with shoulder pathology-a systematic review and meta-analysis," Rheumatology (United Kingdom), vol. 52, no. 4, pp. 743-751, 2013. 


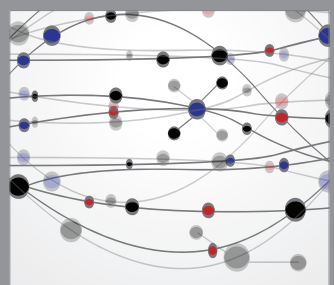

The Scientific World Journal
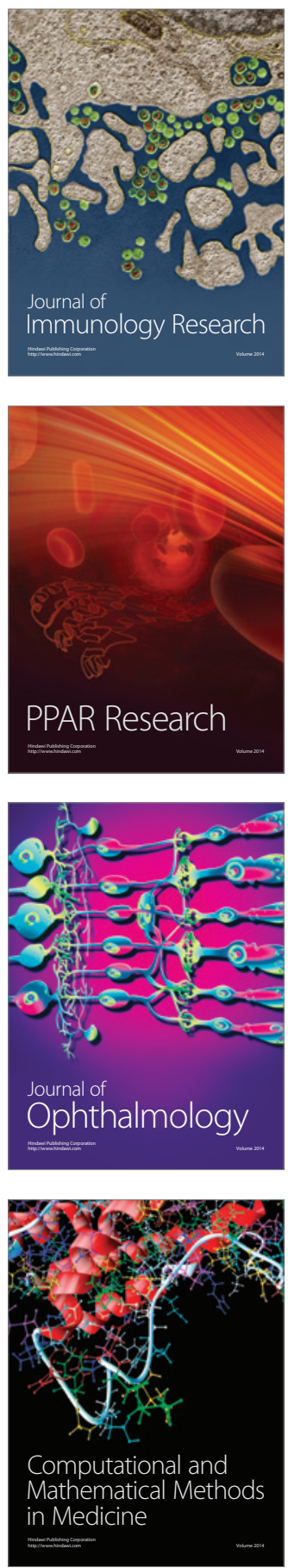

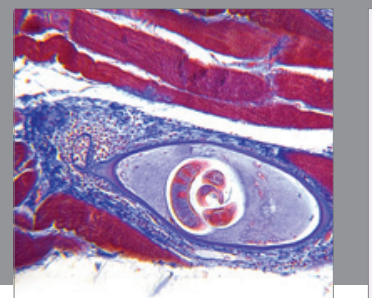

Gastroenterology

Research and Practice
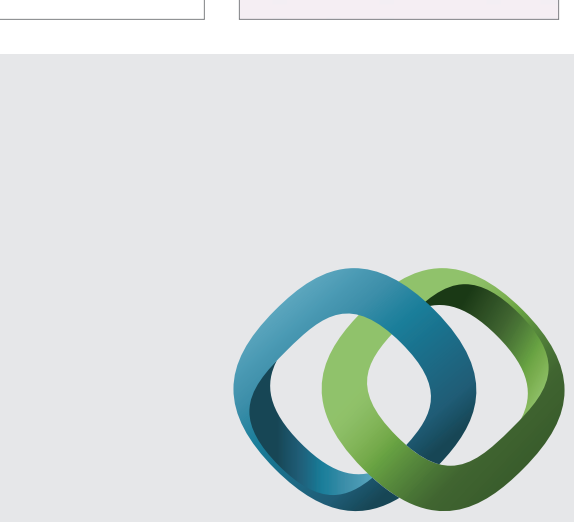

\section{Hindawi}

Submit your manuscripts at

http://www.hindawi.com
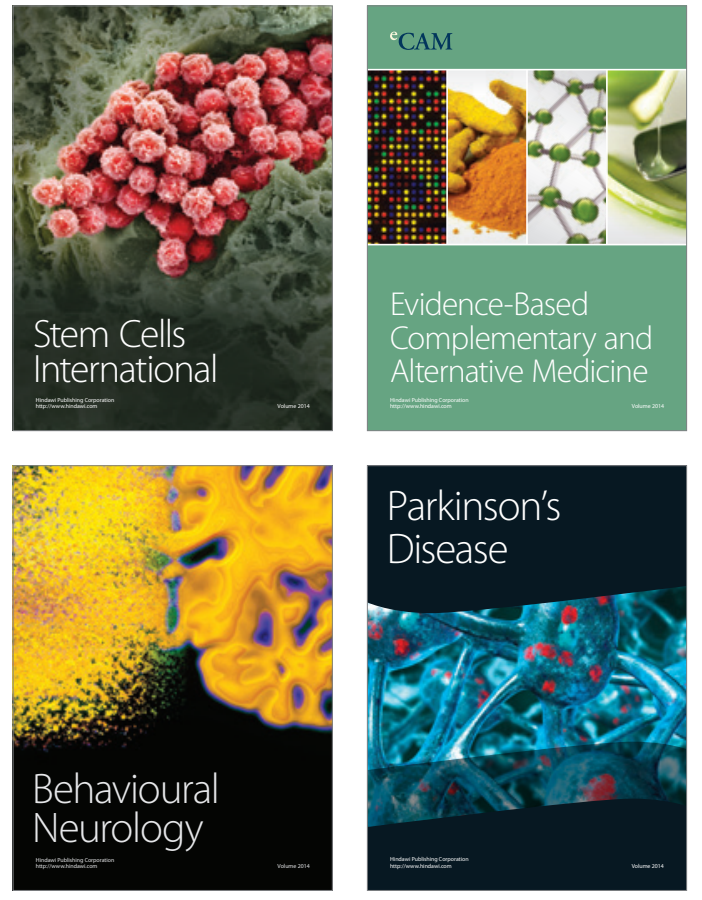
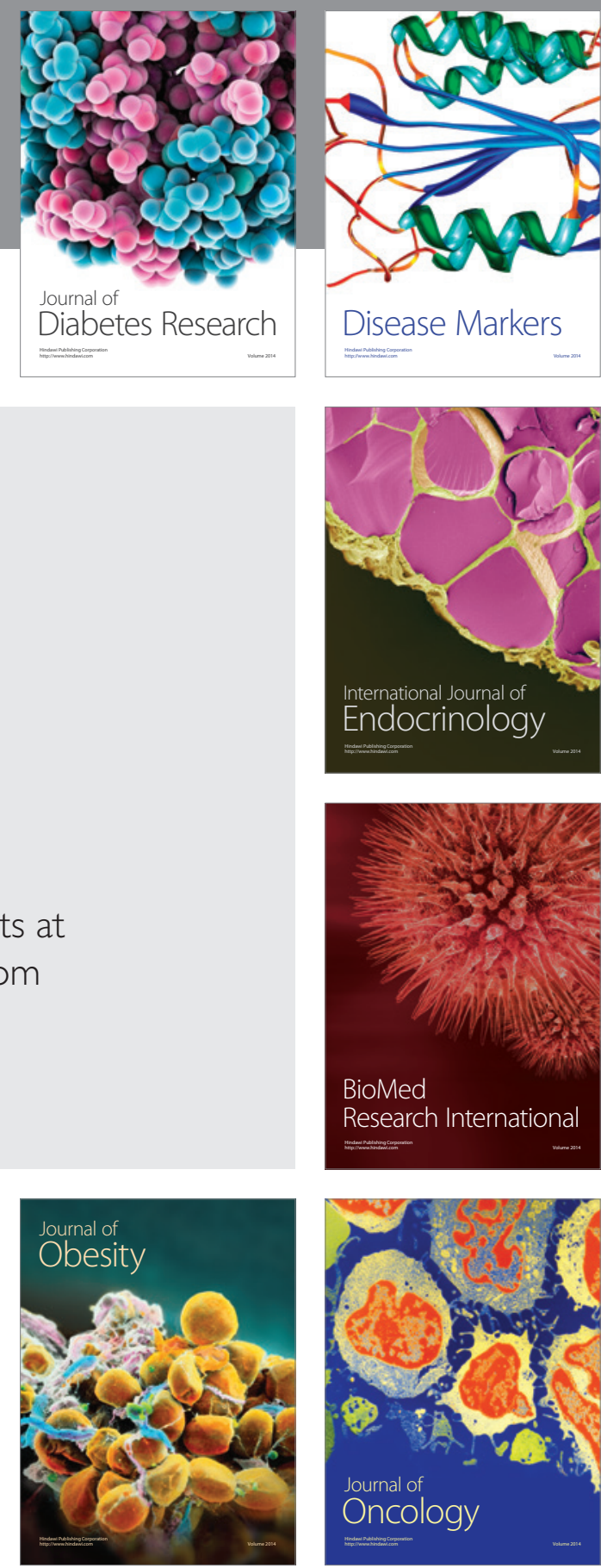

Disease Markers
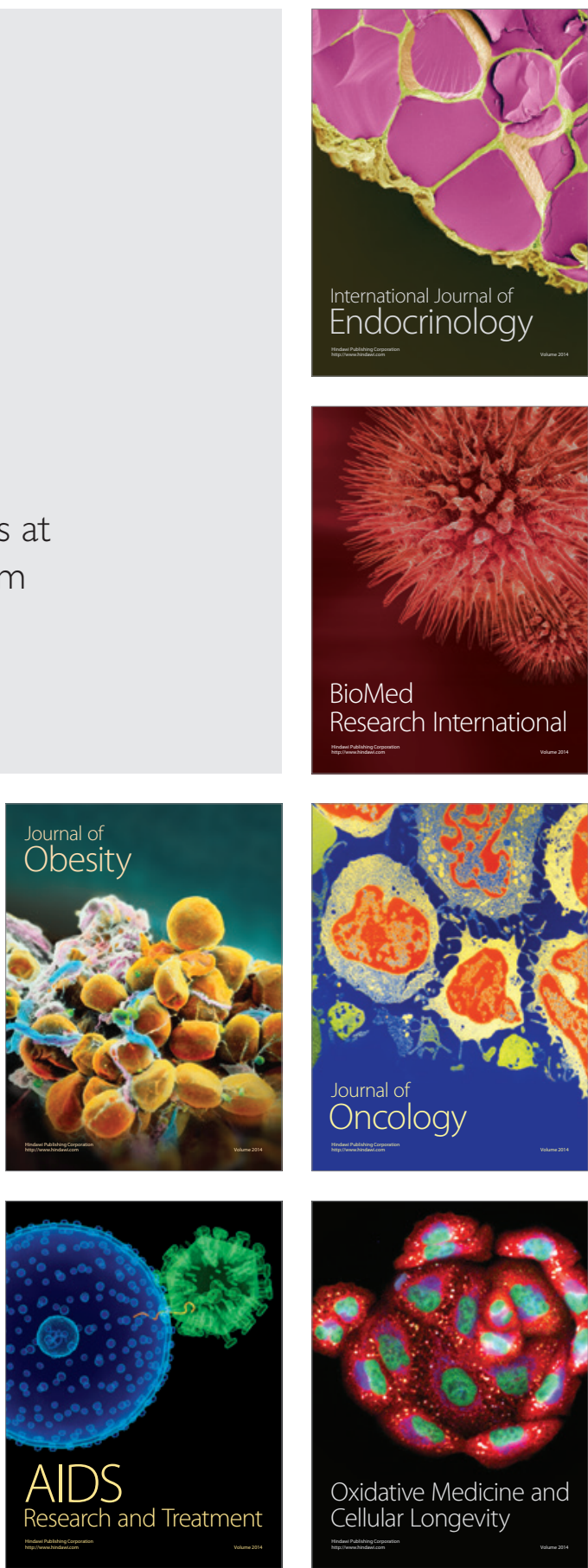